\title{
Enucleation of large radicular cyst and regeneration of bone using autogenous bone marrow cells
}

\begin{abstract}
A large periapical cyst in the right maxillary sinus region with expansion of the medial, anterior and posterior walls of the sinus as well as expansion of the buccal and palatal cortical plates, enucleation of the cystic lesion was performed and regeneration of the Bone using the patient's bone marrow (hematopoietic) cells was done. Achieved results were above satisfactory level. The interesting point to view and understand in this case report is not the pathologic lesion described in the case presentation but the positive results that we have achieved by the process of Regenerative Stem Cell Therapy using autogenous bone grafting techniques. Regenerative Stem Cell Therapy using our patient's bone marrow cells proved to be successful in this case, adequate bone regeneration was seen 1year post operatively in the right posterior maxilla as well as in the inferior wall of the maxillary sinus.
\end{abstract}

Volume 9 Issue 5 - 2018

\author{
Sachin Patel, Mukul Padhye, Punit Shah \\ Department of Oral and Maxillofacial Surgery, Dr. D.Y. Patil \\ Dental College and Hospital, India
}

Correspondence: Sachin Patel, Department of Oral and
Maxillofacial Surgery, Dr. D.Y. Patil Dental College and Hospital,
Sector 7, Nerul Navy Mumbai, India, Tel +I (909)6704700, Email dr.sachinppatal@gmail.com

Received: February 23, 2018 | Published: October 19, 2018

\section{Introduction}

Enucleation of a large $5 \times 5$ centimeter radicular cyst was performed on our patient and infected alveolar bone specifically in the right posterior maxillary region as well as infected bone from the walls of the maxillary sinus were curetted. ${ }^{1}$ Regeneration of the bone that was removed proved adequate with a technique known as Regenerative Stem Cell Therapy using autogenous bone grafting techniques, a procedure which is still in its infancy, ${ }^{2}$ and is presently being utilized quite frequently in many different cases.

\section{Case presentation}

A 38-year old 150 pound patient came into the oral surgery department with no known medical history of trauma, diabetes, heart disease or bleeding disorders and the patient appeared to be in healthy condition. The patient presented with a chief complaint of an asymptomatic swelling in the right maxillary region for the past 14years. The Patient noticed the swelling 6months back with cloudy nasal discharge from the right nostril. On examining the patient the swelling could be palpated in the right maxillary region and was found to be hard in its consistency with sharp rounded borders. ${ }^{3}$ Upon examining intra orally a large palatal swelling was seen measuring $5 \times 5$ centimeters and extending from tooth numbers 2 to 10 (universal system), the teeth that were involved were numbers 4 and 5 in which incipient caries were noticed. A Posteroanterior Skull view radiograph was taken which showed buccal and palatal expansion of the cortical plates. A Cat Scan was taken and on an intravenous contrast Cat Scan taken with an Axial view at the level of the ramus of the mandible and maxillary sinus showed an expansile radiolucent lesion in the right maxillary sinus involving the whole sinus cavity with expansion of the medial, anterior and posterior walls. ${ }^{4}$ The lesion also shows encroachment of the nasopharynx without break in the continuity of the sinus walls (Figure 1).

\section{Discussion}

We know that hematopoietic stem cells contain an abundant source of cells, ${ }^{5}$ when harvested from bone marrow could potentially regenerate different tissue types such as osseous tissue, neural tissue, cardiovascular tissue and many more and the same goes for embryonic stem cells, umbilical cord cells as well as dental pulp cells. ${ }^{6}$ Stem cell research and treatment are still in its infancy but with the continuous improved prognosis on patients it takes us one step closer to the success of Regenerative Stem Cell Therapy. ${ }^{7}$ A radicular cyst is also known as a peri-apical cyst and is a fairly common cyst of the oral cavity as a result of pulpal necrosis due to trauma or severe caries of a tooth. In our case the cause of this cyst was two grossly decayed teeth and the cystic lesion was very large (Figure 1). ${ }^{8}$ Our patient presented to us after 14years of his initial complaint, he had caries on tooth numbers 4 and 5 and had experienced nasal discharge for the last 6 months, which brought him to the department. An odontogenic infected radicular cyst of this nature and size would have to be surgically excised under general anesthesia. In our patient infected bone and tissue, specifically the alveolar bone of the right posterior maxillary region as well as the inferior wall of the maxillary sinus have been curetted and the surgical site was then packed and closed (Figure 2A and Figure 2B). ${ }^{9}{ }^{10}$ Due to the size of the cyst and extent of expansion of the buccal and palatal cortical plates it was safe to assume that the healing of the hollow space would have not been adequate and would cause a large hollow defect in the area of injury post operatively and in the future. So what we advised to the patient was of this procedure called Regenerative Stem Cell Therapy to aid and observe if the healing of bone and the hollow space would be adequate and quick. ${ }^{11}$ After obtaining consent from the patient, bone marrow was extracted from the right iliac crest region, the hematopoietic stem cells were expanded on a specialized medium infused with insulin like growth factors in an incubator at optimal temperature which took approximately 4 weeks, a bio-complex was formed and then we took that bio-complex and injected it with a scaffold as well as a biological membrane into the site of the injury and finished with primary closure. The patient was put on medication, which consisted of initial doses of Intravenous Augmentin 5ml 600mg and Intravenous Diclofenac Sodium and after discharge oral Antimicrobial Tablet Augmentin 875milligrams 1in every 12 hours, Tablet Metronidazole 500mg 1 in every 6hours, and Non-steroidal anti-inflammatory drugs Tablet Diclofenac Sodium1 in every 8hours for pain management. Postoperative radiographs and CAT Scans were taken immediately after surgery (Figure 2A \& Figure 2B) as well as 1year after the surgery (Figure $3 \mathrm{a} \& 3 \mathrm{~b}$ ). Post Operative CT Scans 1year after the surgery showed adequate bone regeneration in the buccal and palatal areas as well as regeneration of the inferior wall of the maxillary sinus (Figure 3A \& Figure 3B [Circled in Red]). There were no post operative complications seen or recorded when the patient was recalled after 1year. 


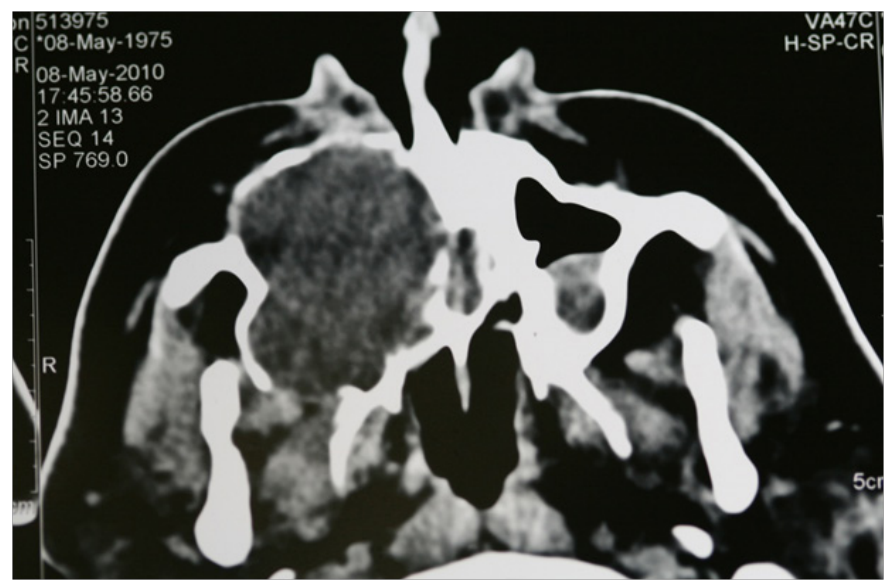

Figure I Pre Operative CAT scan showing large radiolucency in right maxillary sinus region with widening of the superior, inferior, medial and lateral walls of the sinus, as well as encroachment of the nasopharynx.

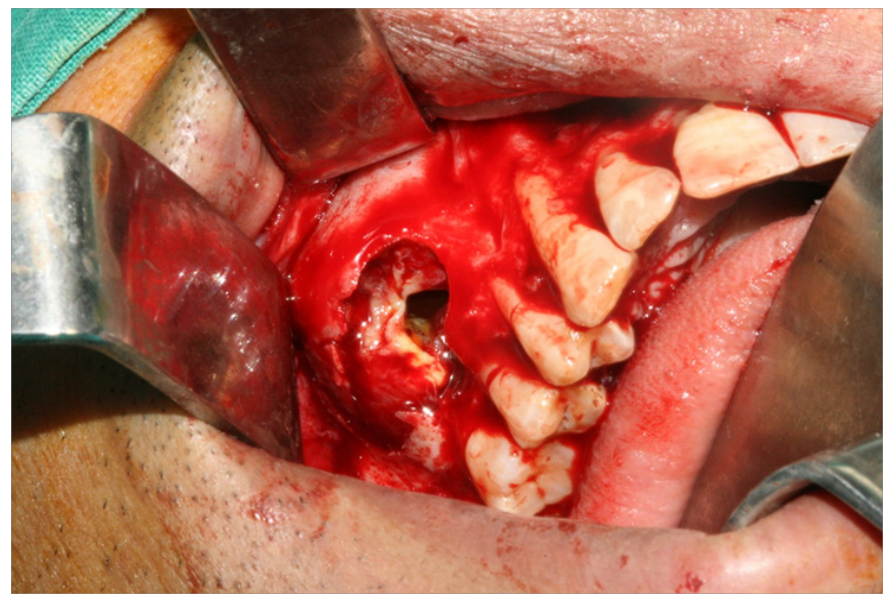

Figure 2 Intra oral operative photo of cystic lesion before enucleation.

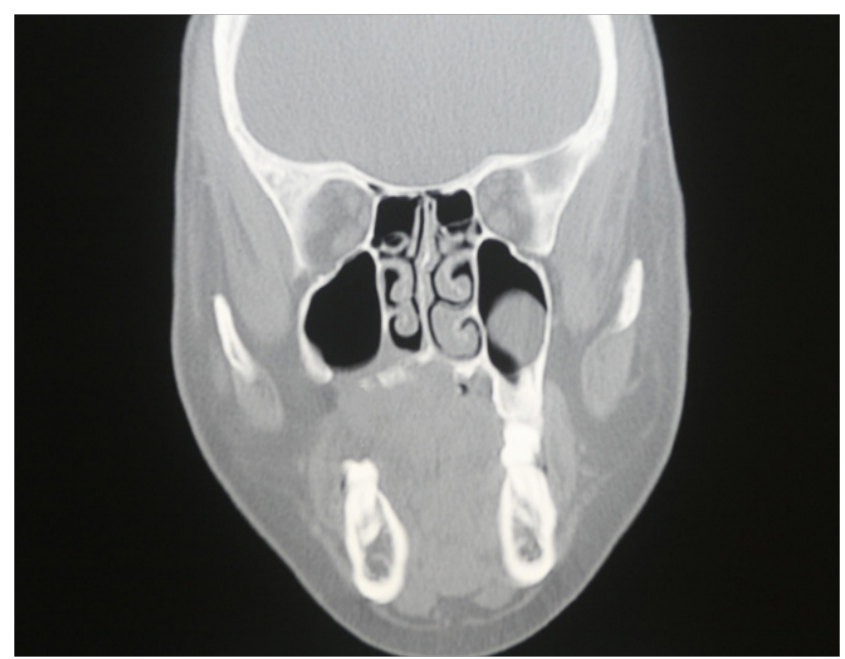

Figure 2A Immediate post operative CAT scan Showing that the cystic lesion has been enucleated and the Inferior wall of the right maxillary sinus and maxillary right posterior alveolar crest bone has been Curetted.

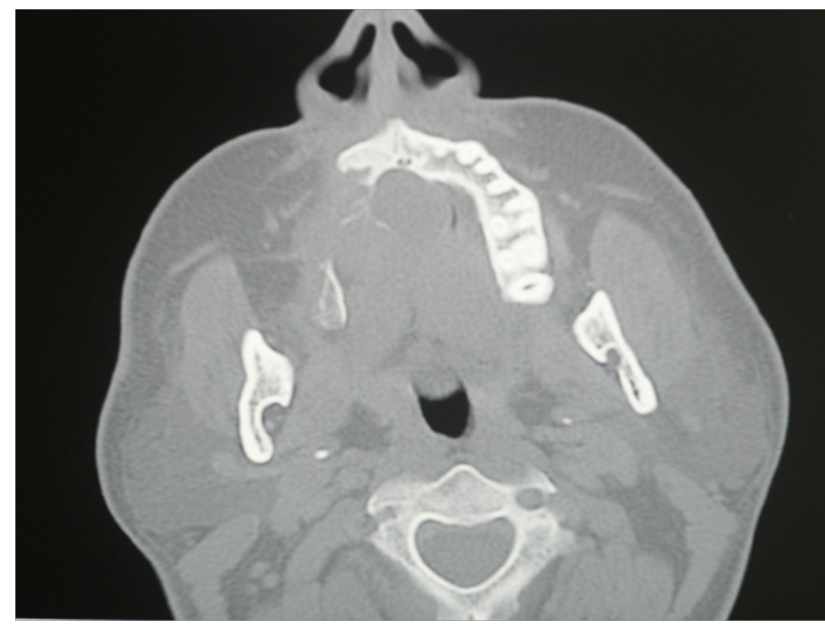

Figure 2B Immediate post operative CAT scan showing the maxillary right posterior alveolar crest bone has been curetted.

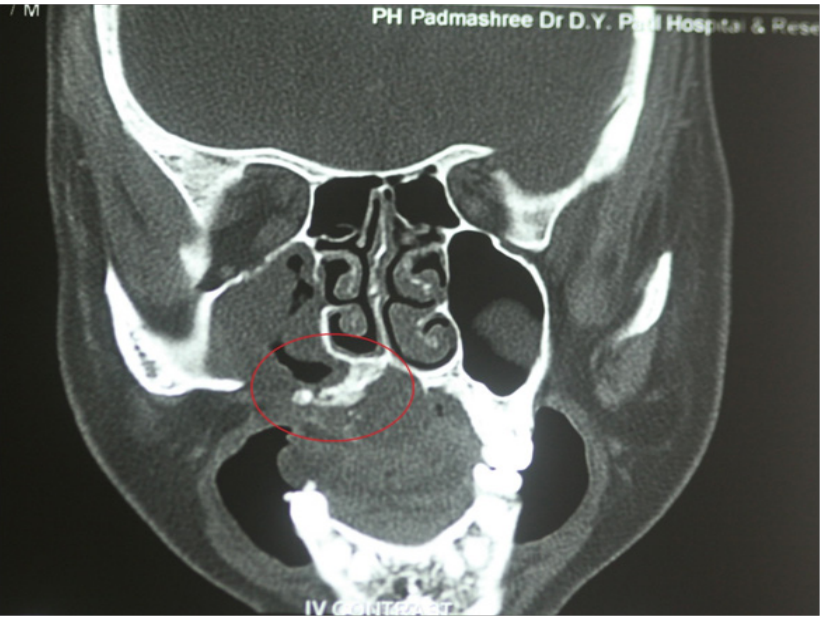

Figure 3A I Year Post Operative CAT Scan showing regeneration of inferior wall of the right maxillary sinus (circled in red).

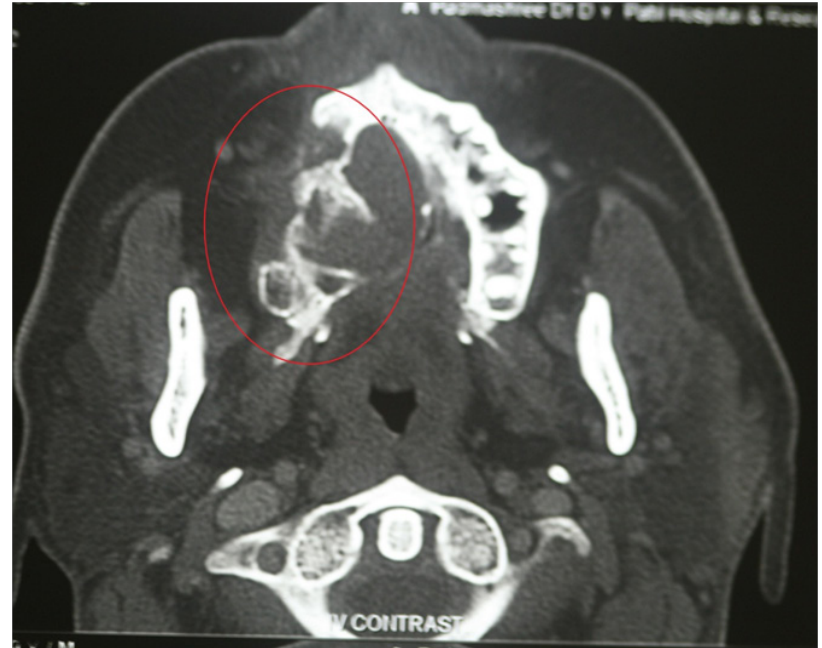

Figure 3B I Year post operative CAT scan showing regeneration of maxillary right alveolar crest bone (Circled in Red).

Citation: Patel S, Padhye M, Shah P. Enucleation of large radicular cyst and regeneration of bone using autogenous bone marrow cells.J Dent Health Oral Disord Ther. 2018;9(5):428-430. DOI: 10.15406/jdhodt.2018.09.00420 


\section{Conclusion}

We can conclude from this 1 patient that Regenerative Stem Cell Therapy with stem cells harvested from the bone marrow of a patient and auto-implanted back into the same patient in the area of operation shows that this procedure can/might be an alternative choice for a patient who may suffer from similar diseases which would require reconstruction/regeneration. The use of stem cells to regenerate bone tissue is a key feature in stating the fact that stem cells do have the ability to form into different cells types, furthermore auto-implantation would less likely cause autoimmune rejection of the graft.

\section{Acknowledgements}

None.

\section{Conflict of interest}

The author declares no conflict of interest.

\section{References}

1. White and Pharoah Textbook of Oral Radiology. 5th ed. Chapter 1920 Inflammatory lesions of the jaw and Cysts of the jaw. EL Sevier Publication; 2004. p. 366-385.

2. Perry D. Patients' voices: the powerful sound in the stem cell debate Science. 2000;287:1423.
3. Endodontics I Oliet, Seymore II Del Rio, Carlos E.

4. Neelima Anil Malik. Textbook of Oral and Maxillofacial Surgery. 2nd ed. Chapter 35. ISBN 81-8448-157-8.

5. Alhadlaq A, Mao JJ. Mesenchymal stem cells: isolation and therapeutics. Stem Cells Dev. 2004;13:436-448.

6. Marion NW, Mao JJ. Mesenchymal stem cells and tissue engineering. Methods Enzymol. 2006.420:339-361.

7. Prusa AR, Hengstschlager M. Amniotic fluid cells and human stem cell research: a new connection. Med Sci Monit. 2002;8:RA253-257.

8. Nair PN, Henry S, Cano V, et al. Microbial status of apical root canal system of human mandibular first molars with primary apical periodontitis after “one-visit” endodontic treatment. Oral Surg Oral Med Oral Pathol Oral Radiol Endo. 2005;99:231-252.

9. Oames JV, Southam JC. Cysts of the jaws and oral soft tissues. Oral Pathology. 3rd edition. In: Soames JV, Southam JC, editors. Oxford: Oxford University Press; 1999:73-92.

10. Chaine A, Pitak-Arnnop P, Dhanuthai K, et al. An asymptomatic radiolucent lesion of the maxilla. Clear cell odontogenic carcinoma. Oral Surg Oral Med Oral Pathol Oral Radiol Endod. 2009;107:452-457.

11. Biology of Blood and Marrow Transplantation. 2012;18(12):1776-1784. 